\title{
Conceptualizing "access" of maternal health services in lower-middle-income countries
}

\author{
Bismah Jameel ${ }^{1}$, Aeda Bhagaloo², Khadija Rashid ${ }^{3}$, Umair Majid ${ }^{4,5 *}$ \\ ${ }^{1}$ Global Health Department, McMaster University, Hamilton, ON, Canada \\ ${ }^{2}$ Centre for Critical Development Studies, University of Toronto Scarborough, Toronto, ON, Canada \\ ${ }^{3}$ Interdisciplinary Centre for Health \& Society, University of Toronto Scarborough, Toronto, ON, Canada \\ ${ }^{4}$ Institute of Health Policy, Management, and Evaluation, University of Toronto, Toronto, ON, Canada \\ ${ }^{5}$ Toronto General Research Institute, University Health Network, Toronto, ON, Canada \\ *Author for correspondence (umair.majid@mail.utoronto.ca)
}

\begin{abstract}
:
A considerable number of women die each year worldwide due to preventable causes during pregnancy and childbirth. The high mortality of women who die from pregnancy-related issues can be attributed to inadequate access of high-quality maternal health services. However, access is a nebulous concept with a compendium of conceptualizations and definitions. In this paper, we discuss the various conceptualizations of access to maternal health services in lower middle-income countries, and compare how issues related to access differ between high- and lower middle-income countries. This discussion informs two priorities that we suggest for researchers conducting health system improvement work in lower middle-income countries: 1) develop a robust understanding of the barriers to access that continue to persist due to cultural, socioeconomic, and political factors, and 2) formulate frameworks and theories specific to lower middle-income countries to guide research.
\end{abstract}

\section{Introduction}

More than 300,000 women die each year worldwide due to preventable causes during pregnancy and childbirth [1]. A significant portion of these deaths can be attributed to inadequate access of high-quality maternal health services, leading to hemmorrhage, hypertensive disorders, sepsis, and deaths through abortion [2]. This is particularly the case for low- and certain middle-income countries, and where the lack of adequate access to maternal health services has slowed cultural and socioeconomic progress.

The concept of health service access serves as a springboard for designing interventions, strategies, and policies to reduce maternal mortality. However, access has a variety of synonyms and definitions, making it difficult to operationalize consistently. In this article, we discuss the various conceptualizations of access in the context of maternal health services. We provide a definition of access applicable for lower middle-income countries - based on the World Bank Atlas method of having a gross national income between $\$ 1026$ and $\$ 3995$ [3] - and describe an example where this definition has been useful for research. We then examine how barriers to access differ between lower middle-income and high-income countries, and how this understanding informs intervention design and delivery around the world. We conclude this paper with considerations for future research on maternal health service access in lower middle-income countries.

\section{Access}

\section{Conceptualization of access}

Gulliford et al. described four dimensions of access: service availability, utilization of services, relevance and effectiveness, and equity. This conceptualization is helpful for developing a common language of access that can support more consistent and coherent research and intervention design [4]. However, there are a number of issues with this conceptualization. First, this framework was published in 2002 and barriers to accessing health services have evolved considerably through the 21st century. Political, educational, and cultural institutions have adapted to changing environmental and social circumstance such as climate change and social unrest. While Gulliford's work may offer important insight into issues with health service access today, it is necessary to develop a framework that accurately reflects contemporary situations. Second, Gulliford's work did not specify application for lower middle-income countries; these countries experience very different political, cultural, and infrastractural issues than low- and high-income countries. A definition of access applicable for low-income countries must be tailored to the circumstances of the country. Third, Gulliford's work was developed through reflections by a handful of experts; however, there is a considerable amount of literature on access that provides a better and more accurate picture of the barriers to accessing mater-
This is an Open Access article distributed under the terms of the Creative Commons Attribution License (CC BY 4.0)

License deed can be found at: http://creativecommons.org/licenses/ by $/ 4.0 /$ https://doi.org/10.29173/hsi286

Received 20 January 2020 Accepted 28 June 2020 
nal health services. We believe a framework that defines and measures access using a systematic evidence synthesis methodology might offer more important insights [5].

There are also other conceptualizations of access. For example, Taylor et al. defined access to medical care as a social indicator of the "processes and outcomes of individuals' passage through the medical system" [6]. Oliver et al. described equity to access health care by providing equal opportunities for accessing health care to those with equal needs, but unequal opportunities to those with unequal needs [7].

More recently, Jacobs et al. developed a framework for addressing access barriers to health services in low-income countries in Asia [8]. They combined two previously published frameworks to list supply-side (i.e., health system) and demand-side (i.e., patient, family, and community) barriers under four dimensions of access: geographic accessibility, availability, affordability, and acceptability. Most notably, the authors assert that their framework aids in identifying the barriers to accessing maternal health services and developing interventions that can address these barriers [8]. The framework and review of interventions were applied to two case studies in Cambodia to show the utility and applicability of this framework for research and intervention design.

We used this framework in a systematic review and framework analysis of 22 studies discussing the barriers to access maternal health services in South Asian countries (Afghanistan, Bangladesh, Bhutan, India, Maldives, Nepal, Pakistan, Sri Lanka) [9]. We have used the framework items as categories of different barriers that organized our analysis of the included literature. For example, we counted the frequency of times "community and cultural preferences" (demand-side barrier for the accessibility dimension) appeared in the included literature, as well as the underlying meaning of how community and cultural preferences became a barrier to accessing maternal health services. In some studies, it was the husband instructing their wives to not seek maternal health care [10]. In other studies, mothers were expected to prioritize their family's needs over their own [11].

Overall, with the exception of certain framework items such as "community and cultural preferences", "staff interpersonal skills" (supply-side barrier for the accessibility dimension), "unqualified health workers, staff absenteeism, opening hours" (supply-side barrier for the availability dimension), and "education" (demand-side barrier for the availability dimension), we have found limited utility of this framework for two reasons. First, the framework items do not comprehensively represent the barriers to maternal health service access that we have encountered in our work. For example, we have found multiple instances where community empowerment and engagement were present in included articles. However, the framework is unclear whether and how the lack of community empowerment and engagement can be a demand-side barrier to maternal health service access. There is also a need to expand the framework to be better applicable to current research, as well as the structure and function of health systems in Asia.
Second, we have found the description of framework items to be inadequate to use as a tool for investigating the barriers to accessing maternal health services. For example, does "education" (demand-side barrier to the availability dimension) include elements of "lack of health awareness" (demand-side barrier to the acceptability dimension)? Does "education" incorporate accessibility and availability of interventions that aim to improve knowledge about pregnancy and childbirth? We suggest researchers who are considering using this framework for research to first formulate consistent definitions that are applicable to their topic and research questions. Overall, this section illustrates that although there are definitions and conceptualizations of access, there are important challenges in using them for research and intervention design.

\section{Access in lower middle-income vs. high-income countries}

There are notable distinctions between high-income and lower middle-income countries in the structure and function of health systems. These distinctions lead to differences in the challenges and barriers individuals may face when accessing maternal health services. Therefore, using the access framework from Jacobs et al., we have outlined the most pertinent distinctions between high-income and lower middle-income countries that are important for studying health service access in Table 1 [8].

\section{The design and delivery of maternal health interventions in lower middle-income countries}

Interventions designed by educational or research institutions play a crucial role in delivering high-quality maternal health services in low-income communities. We recently published a scoping review of the design and delivery of maternal health interventions in Pakistan [12]. We analyzed 23 articles describing maternal health interventions for their intervention characteristics in Pakistan only. This knowledge provided a snapshot of the work that has been conducted in Pakistan, and priorities for future intervention design, delivery, and research. For example, we recommended developing multi-level and multi-component interventions to more efficiently improve maternal health care outcomes. At the same time, studies need to be transparent on if and how they involved stakeholders in the design and delivery of interventions, as well as the potential impacts of their approach to stakeholder engagement. Finally, there were more interventions without a formal evaluation or with evaluation that was planned but not published. This finding may indicate that although there are increasing efforts to design maternal health interventions, these efforts may have been interrupted prematurely due to lack of consistent funding and support.

More importantly, although this work was conducted 
Table 1 | Overview of the major differences between lower middle-income and high-income countries that influence health service access

\begin{tabular}{lll}
\hline Characteristic & Lower Middle-Income Countries & High-Income Countries \\
\hline Evidence-based culture & $\begin{array}{l}\text { An emerging, but weak and fragmented evi- } \\
\text { dence-based culture; this limits the quantity } \\
\text { and quality of research evidence available on } \\
\text { these countries. }\end{array}$ & $\begin{array}{l}\text { Presence of a strong evidence-based culture that } \\
\text { promotes the production and use of knowledge. }\end{array}$ \\
\hline Transportation Infrastructure & $\begin{array}{l}\text { A developing transportation infrastructure, } \\
\text { primarily for urban areas. Rural areas contin- } \\
\text { ue to be largely inaccessible in the majority of } \\
\text { countries. }\end{array}$ & $\begin{array}{l}\text { Strong transportation infrastructure in all urban } \\
\text { areas and most rural areas that limit issues of } \\
\text { geographic accessibility. }\end{array}$
\end{tabular}

\begin{tabular}{ll}
\hline Universal Health Coverage & Although an increasing number of countries \\
have elements of universal health care, there \\
remain important gaps.
\end{tabular}

Medical Education Medical education and training are medium to high-quality. However, these countries are challenged with "brain-drain" where expert healthcare providers immigrate to high-income countries.

The majority of countries have some form of a universal health coverage insurance scheme, either through the government or private employers.

Medical education and training are of consistently high-quality. There are also multiple mechanisms to ensure health workers provide high-quality care (e.g., performance monitoring).

Information Access Access to essential information on health services is increasing, as well as consistent internet access.

Information on health services, government programs, and educational interventions is easily accessible via internet or local healthcare providers.

Health Awareness Campaigns

There are a number of educational health awareness campaigns in these countries, but information does not reach the majority of people, and the culture on prioritizing personal health is still developing

Patient Autonomy
Although changing, there is still a considerable amount of paternalism in health care, limiting the extent to which patient can voice their preferences and challenge healthcare providers.
Educational health awareness campaigns reach a greater number of individuals because of a strong culture on personal health and well-being.

There has been a rapid shift in patient preferences to be more involved in their clinical care. This observation reflects an increase in self-esteem and confidence to speak to and challenge healthcare providers. on Pakistani studies, it illustrated a mismatch between policy and practice that may be promulgating the barriers to maternal health service access in lower middle-income countries. For this reason, we believe that there is a need to formulate conceptualizations and frameworks of access on how a mismatch between policy and practice influences the barriers to access that persist. Having conducted this scoping review, we are working on a systematic review and content analysis of Pakistani policy documents to better understand the pathway from government and organizational priorities to the implementation of interventions as well as how this pathway can be optimized.

\section{Future directions for research on maternal health services in lower middle-income countries}

Based on our discussion, we suggest two steps for future research on maternal health service access in lower middle-income countries, particularly in Asia.

\section{Develop a robust understanding of the barriers to access that continue to persist due to cultural, socioeconomic, and political factors}

Although there continues to be a need to develop new interventions in lower middle-income countries, it is imperative to better understand the factors that influence their delivery in different communities, belief systems, and circumstances. For this reason, our work steps back from the admirable work by organizations and the government that addresses specific maternal health indicators in South Asian countries. Instead, our work underlies intervention design with the socio-cultural factors that determine whether or not an intervention will have its intended effect when implemented in the real-world and on a larger scale. We are also conducting several targeted reviews for understanding the barriers that exist in different regions in Asia, and how they compare with each other. Our goal from this work is to initiate discussion on the most effective and appropriate strategies that lead to sustainable improvement in maternal health. 


\section{Formulate frameworks and theories specific to low- and middle-income countries to guide research in South Asia}

As we have demonstrated in this paper, the issues to accessing maternal health services differ considerably between lowand high-income countries. Notwithstanding, we have seen frameworks and theories that were developed and tested in high-income contexts be inappropriately applied to health care issues in lower middle-income countries and contexts. We believe that this may be in part why the widespread efforts to improve maternal health services have not observed the predicted successes. It may be more appropriate and effective to enable individuals in these countries, who best understand the socio-cultural and political environments, to formulate theories and frameworks best suited for research in their country. Therefore, we suggest researchers from high-income countries to focus on strengthening the capacity for researchers residing in lower middle-income countries to tailor theories and interventions for their contexts.

\section{Limitations of this commentary}

There are a number of limitations in this paper. First, we focus solely on lower middle-income countries; we believe that the literature on low-income countries might offer important insight on issues with accessing maternal health services. Second, the evidence syntheses discussed in this paper are specific to certain South Asian countries. Since South Asian countries face very different cultural and political issues compared to each other, they may also have diverse challenges with accessing health services. While we did not focus on exploring these issues in this paper, we believe that they are a direction for future research.

\section{Conclusion}

In this paper, we discussed the various conceptualizations of health service access in lower middle-income countries, and compare how access issues differ between high- and lower middle-income countries. We suggest two priorities for researchers conducting health system improvement work in lower middle-income countries: 1) develop a robust understanding of the barriers to access that continue to persist due to cultural, socioeconomic, and political factors, and 2) formulate frameworks and theories specific to lower middle-income countries to guide research.

\section{References}

1. Roser M, Ritchie H. "Maternal Mortality". Published online at OurWorldInData.org. 2019, retrieved from: 'https://ourworldindata.org/ maternal-mortality' [Online Resource]

2. Say L, Chou D, Gemmill A, Tunçalp Ö, Moller AB, Daniels J, Gülmezoglu AM, Temmerman M, Alkema L. Global causes of maternal death: a WHO systematic analysis. The Lancet Global Health. 2014 Jun 1;2(6):e323-33.

3. World Bank Group. The World Bank Atlas method - detailed methodology. 2019, https://datahelpdesk.worldbank.org/knowledgebase/ articles/378832-what-is-the-world-bank-atlas-method

4. Gulliford M, Figueroa-Munoz J, Morgan M, Hughes D, Gibson B, Beech R, Hudson M. What does' access to health care'mean?. Journal of health services research \& policy. 2002 Jul 1;7(3):186-8.

5. Majid U, Zain S, Shaikh R, Jameel B. Uncommon Methodologies for Conducting Literature Reviews: Part 1-A Policy Analysis of How Maternal Health Concepts Have Been Framed in Policy Documents of a Low-Income Country. 2020, SAGE Research Methods Cases

6. Taylor DG, Aday LA, Andersen R. A social indicator of access to medical care. Journal of health and Social Behavior. 1975 Mar 1:3949.

7. Oliver A, Mossialos E. Equity of access to health care: outlining the foundations for action. Journal of Epidemiology \& Community Health. 2004 Aug 1;58(8):655-8.

8. Jacobs B, Ir P, Bigdeli M, Annear PL, Van Damme W. Addressing access barriers to health services: an analytical framework for selecting appropriate interventions in low-income Asian countries. Health policy and planning. 2011 May 12;27(4):288-300.

9. Jameel B, Rashid K, Bhagaloo A, Majid U. Barriers to accessing maternal health services in South Asia: a framework for future inquiry and intervention design. In progress.

10. Biswas A, Dalal K, Abdullah AS, Gifford M, Halim MA. Maternal complications in a geographically challenging and hard to reach district of Bangladesh: a qualitative study. F1000Research. 2016;5.

11. Ahmed S, Khan MM. A maternal health voucher scheme: what have we learned from the demand-side financing scheme in Bangladesh? Health policy and planning. 2010 Apr 7;26(1):25-32.

12. Zain S, Jameel B, Zahid M, Munir M, Kandasamy S, Majid U. The Design and Delivery of Maternal Health Interventions in Pakistan: A Scoping Review. Healthcare for Women International. 2020, in press. https://doi.org/10.1080/07399332.2019.1707833

(C) The Author(s) 